\title{
Coronavirus disease-19 in Hematology. The perspective of a middle-income country
}

\author{
David Gómez-Almaguer*, Luis J. Marfil-Rivera, Olga G. Cantú-Rodríguez, César H. Gutiérrez-Aguirre, \\ Perla R. Colunga-Pedraza, Luz C. Tarín-Arzaga, Andrés Gómez-De León, José C. Jaime-Pérez, and \\ Elsa L. Zertuche-González
}

Hematology Service, School of Medicine and "Dr. José Eleuterio González" University Hospital, Universidad Autónoma de Nuevo León, Monterrey, Nuevo Leon, Mexico

\begin{abstract}
Coronavirus disease (COVID)-19 is an infectious disease affecting mainly the respiratory system; other organs and tissues can also be affected, including the blood. Hematologists are now treating patients that experience COVID-19-associated to pathological hematological findings such as lymphopenia and thrombocytopenia and complications such as cytokine release syndrome, thrombosis, and disseminated intravascular coagulation. Several drugs from hematologist's therapeutic armamentarium have been proposed as candidates for treating patients with COVID-19, including enoxaparin, tocilizumab, ibrutinib, and others. Transfusion of plasma from COVID-19 recovered patients is another biological therapeutic option available. The blood and COVID-19 are a topic which hematologists from low-and-middle-income countries should dominate. We must be creative and learn how to apply the limited resources available to face this paradigm-changing challenge.
\end{abstract}

Key words: Coronavirus disease-19. Hematology. Plasma. Lymphopenia. Blood. Infectious disease.

\section{Introduction}

The world is now facing another new viral pandemic. There is a global outbreak of a novel coronavirus. Coronavirus disease (COVID)-19 is a disease caused by severe acute respiratory syndrome coronavirus 2 (SARSCoV-2). It is now clear that every continent in the world has been affected. The infection has been diagnosed in all age groups, but it is uncommon in children and adolescents. At present, no ideal treatment or specific anti-COVID-19 drugs are available. Therefore, the World Health Organization has focused on infection prevention, detection, and patient monitoring, as in other viral epidemics, treatment options include antivirals, antimalarial, steroids, among other treatments ${ }^{1}$. The efficacy of most of these drugs is still unknown, with supportive care being of utmost importance. Patients with COVID-19 present with clinical symptoms frequently have hematologic abnormalities. Some therapies common to the hematological field have been proposed as potentially useful candidates in the fight against COVID-19 due to a variety of mechanisms. Therefore, in this review, a group of Mexican hematologists from a single academic institution discuss several concepts and ideas according to the information currently available for managing patients with COVID-19.

\section{Correspondence:}

*David Gómez-Almaguer

E-mail: dgomezalmaguer@gmail.com
Date of reception: 02-07-2020

Date of acceptance: 07-08-2020 DOI: 10.24875/RMU.20000062
Available online: $30-10-2020$ Medicina Universitaria. 2020;22(3):120-126 www.medicinauniversitaria.org

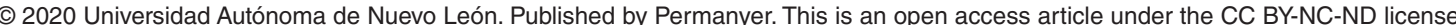
(http://creativecommons.org/licenses/by-nc-nd/4.0/). 


\section{Lymphopenia}

A frequent laboratory finding in patients infected with COVID-19 is lymphopenia. Although the total white blood cell count may be normal or slightly decreased, lymphopenia is generally present. An absolute lymphocyte count $<1 \times 10 \%$ has been associated with severe disease $^{2}$. In one of the first articles published, Liu et al. analyzed 12 patients with COVID-19, finding lymphopenia in $50 \%$ of cases, correlating higher viral load with a higher degree of lymphopenia $(r: 0.7, p=0.01)$ the total decrease in lymphocytes ( $r: 0.77, p=0.003$ ) and their percentage ( $r: 0.6, p=0.01$ ) with more severe lung injury, when it was evaluated by the Murray scale ${ }^{3}$. Other authors in studies with a greater number of patients $(n=41)$ reported similar data: an overall incidence of lymphopenia (lymphocytes $<1 \times 10^{9} / \mathrm{L}$ ) of $63 \%$ observing that $85 \%$ of patients admitted to intensive care had lymphopenia 4 . In patients with severe disease, a decrease in CD4+ T lymphocytes is mainly observed (Table 1). It has been hypothesized that CD4+ T lymphocyte count could be used as a predictor of severity and prognosis, as survival may be associated to the ability to replace the lymphocytes removed by the virus. In a study that included 93 patients with COVID 19 (24 with severe disease), lymphopenia and neutrophilia were observed in $80.6 \%$ and $51.6 \%$, respectively. This contrast, explained by the authors, is presented in response to the inflammatory factors related to the virus (interleukins [IL] 6 and 8, tumor necrosis factor, etc.) that causes neutrophilia and systemic inflammation which suppresses cellular immunity and the number of CD4+ T cells, increasing the neutrophil/lymphocyte ratio (NLR). When the NLR is $\geq 3.3$, $46 \%$ of patients with moderate disease progressed to severe disease in a median of 6.3 days, while patients with NLR $\leq 3.3$ evolved favorably and were discharged in 13.5 days $^{5}$. Although the number of patients included in these studies is limited, lymphopenia in patients with COVID 19 may be an important predictive biomarker, potentially assisting decision-making. The absolute lymphocyte count is a highly attractive biomarker for hematologists in low- and middle-income countries (LMICs) as it is an inexpensive and widely available parameter. In the very near future, we will hopefully see the publication of studies with a larger number of patients and will be able to define this behavior.

\section{Hemostasis}

COVID-19 can occur with a wide variety of expressions ranging from an asymptomatic state to multiple
Table 1. Relevant hematological findings in patients with COVID-19

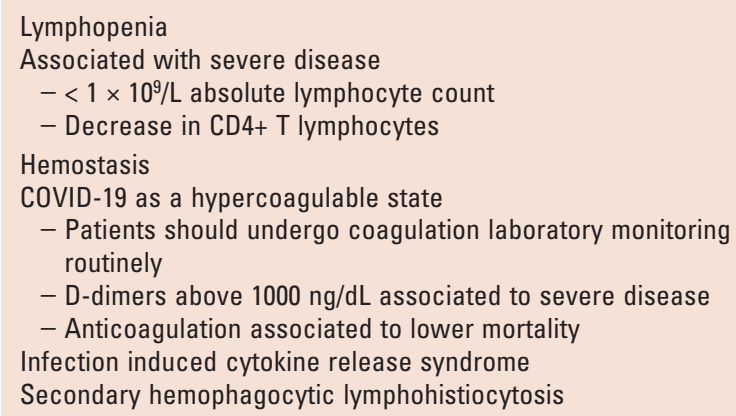

organic dysfunction syndrome and death. COVID-19 can also show manifestations of coagulation dysfunction. Just like in other critically ill patients, the host inflammatory reactions can culminate in an increased production of pro-inflammatory cytokines which result in high levels of IL-6, C-reactive protein, and fibrinogen, and also because of the tropism the virus has for angiotensin-converting enzyme 2 receptors, resulting in endothelial cell damage and impairment of the natural antithrombotic state ${ }^{6}$.

The proposed mechanism to reach this serious complication is multifactorial, including a hypercoagulable state (thrombophilia) with the presence of thrombosis mainly at the level of pulmonary microcirculation, although it can affect the whole body. Other manifestations associated with a hypercoagulable state are the presentation of pulmonary thromboembolism, stroke, myocardial infarction, and peripheral venous thrombosis $^{6,7}$. Coagulation dysfunction is one of the major causes of death in patients with COVID-19, which can present itself with a sepsis-induced coagulopathy, and in some cases, it can evolve to disseminated intravascular coagulation (DIC). Tang et al. demonstrated in a cohort of 183 patients that $71 \%$ in later stages of the disease, who were hospitalized and presented multiorgan failure, died with a diagnosis of DIC according to the criteria of the international society on thrombosis and hemostasis; non-survivors had significantly higher D-dimer and fibrin degradation product, longer prothrombin time, and activated partial thromboplastin compared to survivors ${ }^{6}$.

The increase in D-dimer, prothrombin time, activated thromboplastin time, and decrease in fibrinogen level and platelets was associated with DIC, which could start between day 7 and 10 after admission. The concentration of D-dimer above $1000 \mathrm{ng} / \mathrm{dL}$ has been shown to be an independent factor predictive of severity ${ }^{8}$ (Table 1). It can be an indirect marker of the increased inflammatory response in this population. The 
theory of immune-thrombosis, where inflammation and thrombin formation are directly correlated, is appealing. Therefore, it has been suggested that heparin could decrease the inflammatory response by preventing the formation of thrombin ${ }^{9}$.

The presence of coagulopathy has led to consider the use of anticoagulant medications as part of the treatment of the disease. The use of an anticoagulant drug appears to be associated with a reduction in mortality, particularly for those patients who have sepsis-associated coagulopathy. Heparins, low molecular weight, unfractionated heparins, and fondaparinux pentamer have been associated with benefits in this group of patients. Heparin can reduce thrombosis in the microcirculation, especially in pulmonary vasculature ${ }^{10}$. A recent meta-analysis reported a reduction in mortality with early administration of low molecular weight heparin (LMWH) in a population of SARS patients with COVID no-19. Finally, heparin may possess antiviral properties by acting on SARS-CoV-2 surface receptor binding proteins and inhibit viral binding ${ }^{9}$ (Table 2).

Clear and solid evidence in this setting is lacking, but the following recommendations are aimed at providing a reasonable and practical approach for specialists and allied health professionals:

- Hospitalized patients with COVID-19 should undergo a thorough laboratory assessment of hemostasis, in particular thrombin peak, time to peak and thrombin, determination of D-Dimers, fibrinogen quantification, platelet count, and fibrinogen degradation products ${ }^{11}$.

- Serial determinations of coagulation studies should be made in all hospitalized patients. The ideal interval has not yet been defined ${ }^{11}$.

- All patients should be placed in a thromboprophylaxis protocol with prophylactic doses of anticoagulation, preferably with LMWH unless there is a contraindication for this ${ }^{10}$.

- Therapeutic anticoagulation should be strongly considered in patients at high risk of coagulopathy, in patients demonstrating signs of thrombosis-induced organic dysfunction in microcirculation, or with documented or strongly suspected thromboembolism ${ }^{11}$.

- Although some reports support the use of infusion thrombolytic drugs as a rescue treatment in refractory cases, evidence of the safety of this practice is lacking $^{12}$.

- Aspirin should only be considered in cases with elevated troponin and cardiac dysfunction ${ }^{13}$.
Table 2. Recommendations for the use of enoxaparin in patients with COVID-19

\begin{tabular}{|c|c|c|}
\hline Patients & $\begin{array}{l}\text { Renal clearance } \\
>30 \mathrm{ml} / \mathrm{min}\end{array}$ & $\begin{array}{l}\text { Renal clearance } \\
<30 \mathrm{ml} / \mathrm{min}\end{array}$ \\
\hline $\begin{array}{l}\text { Patients without major } \\
\text { risk factors of } \\
\text { thrombosis }\end{array}$ & $\begin{array}{l}40 \mathrm{mg} / 24 \text { h SO } \\
80-100 \mathrm{~kg}: 60 \\
\mathrm{mg} / 24 \mathrm{~h} \mathrm{SO} \\
>100 \mathrm{~kg}: 40 \mathrm{mg} / 12 \\
\text { h S0 }\end{array}$ & $\begin{array}{l}20 \mathrm{mg} / 24 \mathrm{~h} \mathrm{~S} 0 \\
>80 \mathrm{~kg}: 40 \mathrm{mg} / 24 \mathrm{~h} \\
\text { S0 }\end{array}$ \\
\hline $\begin{array}{l}\text { Patients with major risk } \\
\text { factors of thrombosis }\end{array}$ & $1 \mathrm{mg} / \mathrm{kg} / 24 \mathrm{~h} \mathrm{~S} 0$ & $0.5 \mathrm{mg} / \mathrm{kg} / 24 \mathrm{~h} \mathrm{SQ}$ \\
\hline $\begin{array}{l}\text { Patients with venous } \\
\text { thromboembolism }\end{array}$ & $\begin{array}{l}1 \mathrm{mg} / \mathrm{kg} / 12 \mathrm{~h} \mathrm{SO} \\
1.5 \mathrm{mg} / \mathrm{kg} / 24 \mathrm{~h} \mathrm{SO}\end{array}$ & $1 \mathrm{mg} / \mathrm{kg} / 24 \mathrm{~h} \mathrm{SO}$ \\
\hline
\end{tabular}

SQ: subcutaneous

\section{Cytokine release syndrome (CRS) and anti-IL-6 antibodies}

After SARS-CoV-2 binds to alveolar epithelial cells, activation of innate and adaptative immune system occurs. This can result in CRS and its most severe form termed secondary hemophagocytic lymphohistiocytosis (sHLH). This is characterized by excessive production of inflammatory cytokines, including IL-1, IL-2, IL-6, IL-7, interferon- $\gamma$, monocyte chemo-attractant protein 1 , macrophage inflammatory protein $1-\alpha$, granulocyte-colony stimulating factor, and tumor necrosis factor- $\alpha^{4}$. IL-6 is considered one of the key cytokines involved in infection-induced CRS and has been shown as an important predictor of severe COVID-194 ${ }^{4}$ A recent systematic review and meta-analysis found significantly higher serum IL-6 levels compared to non-severe patients and increased mortality ${ }^{14,15}$. For this reason, the management of this immune phenomenon with targeted drugs that inhibit IL-6 is being explored. Tocilizumab (TCZ) (Actemra ${ }^{\circledR}$ ) is a recombinant monoclonal antibody against both the soluble and membrane-bound IL-6 receptor, mainly employed in autoimmune diseases such as rheumatoid arthritis ${ }^{16}$. In the field of hematology, TCZ was approved by the Food and Drug Administration since 2017 for the treatment of CRS following chimeric antigen receptor T-cell therapy ${ }^{17}$. Under this premise, blockade of IL- 6 with TCZ has been used as a potential therapy in severe COVID-19 patients displaying signs of CRS. However, debatable results regarding $\mathrm{TCZ}$ effectiveness have been reported in retrospective studies or case reports. Whereas some have reported a significant improvement in the symptoms, oxygenation, and computed tomography opacity changes, others have described harmful 
outcomes after TCZ infusion ${ }^{18-23}$. A retrospective analysis of 21 severe COVID-19 cases treated with TCZ showed a reduction in fever and lung lesion opacity, and peripheral blood lymphocyte count recovery ${ }^{18}$. On the other hand, a case report observed progression to sHLH despite TCZ administration suggesting that excessive IL-6 production may only be a small component of the CRS seen in COVID-1923. Chinese health commission and Italian guidelines support the use of TCZ in severe COVID-1924,25. As other monoclonal antibodies, TCZ administration is not risk-free, and thus its use could be considered to be reserved only for moderate/ severe cases with rapid clinical and/or radiologic worsening. Beneficial effects should be balanced with possibly harmful effects secondary to the inhibition of antiviral immunity that could potentially delay viral clearance. Special precautions should be established ideally before the start of therapy ${ }^{26}$. Classical contraindications to its use comprise transaminases levels $>5$ times the upper limit of normal, neutrophil count $<500$ cells $/ \mu \mathrm{L}$, and platelet count $<50,000$ cells $/ \mu \mathrm{L}^{27}$. At present, 17 clinical trials assessing TCZ in patients with COVID-19 with pending results appear in Clinical Trials.gov registry and a prospective multicenter randomized controlled clinical study led by the first affiliated hospital of University of Science and Technology of China has been recorded in the Chinese clinical trial registry and is expected to be completed soon. Ongoing clinical trials will further elucidate the effect of TCZ on COVID-19-induced organ failure and mortality. Additional agents inhibiting IL-6 and IL-6-mediated signaling might represent therapeutic interventions for COVID-19. Siltuximab is an antibody directed against one of the three distinct binding sites of IL-6 approved in the treatment of multicentric Castleman disease ${ }^{28}$. This agent is currently being evaluated in a Spanish randomized clinical trial (NCT04329650). In conclusion, to date, there is no specific drug for SARS-CoV-2 and cytokine storm induced by this virus and we will have to wait for the results of the current clinical trials to clarify the role of anti-IL-6 drugs.

\section{Drugs}

\section{Janus activating kinase (JAK) inhibitors}

JAK inhibitors limit the activity of one or more JAK enzymes (JAK1, JAK2, JAK2, and TYK3) which normally activate the signal transducer and activator transcription (STAT) proteins. The JAK/STAT pathway is very important in response to cytokine signaling in hematopoiesis and immune cells in health as well as in inflammatory diseases and myeloproliferative neoplasms such as polycythemia vera or myelofibrosis ${ }^{29}$. Potential mechanisms cited to justify their study in COVID-19 include anti-inflammatory properties through blocking of downstream cytokine-induced signaling, suppression of secretory senescent cells and even limiting the entrance of SARS-Cov2 intro pneumocytes, as suggested by theoretical drug target analysis ${ }^{29-32}$. Ruxolitinib, a JAK1/2 inhibitor approved for the treatment of myelofibrosis, polycythemia vera, and acute graft versus host disease, is the JAK inhibitor with the longest track record and widespread availability ${ }^{29}$. Remarkably, despite an almost complete lack of clinical evidence of its safety and efficacy in COVID-19, this highly expensive drug has been made available internationally by the manufacturing company in an expanded access "compassionate use" program for severe disease (NCT04337359) including Mexico. It is currently the focus of multiple clinical trials but particularly an eagerly awaited industry-sponsored, randomized, and placebo-controlled phase III trial entitled RUXCOVID (NCT04362137). Ultimately this drug could also prove harmful to COVID-19 patients as it may hamper the INF-mediated response, theoretically slowing SARSCov2 clearance ${ }^{33}$. Ruxolitinib can also cause anemia and thrombocytopenia, potentially worsening the outcome of critically ill patients and was also associated to increased rates of bacterial and herpes zoster infections in the myelofibrosis registration trials ${ }^{29}$. Fedratinib a selective oral JAK2 inhibitor is the second drug in its class available for the treatment of myelofibrosis and proposed as a potential anti-inflammatory COVID-19 therapy. Interestingly, through its selective JAK2 inhibition, fedratinib does not affect type 1 interferons ${ }^{34}$. No clinical trial on its use in COVID-19 patients has been registered. Adverse effects associated to this drug include anemia and gastrointestinal symptoms and have also been controversially associated to a form of encephalopathy ${ }^{35}$. Similarly, other JAK inhibitors used for atopic dermatitis and rheumatoid arthritis such as baricitinib, tofacitinib, and upadactinib have also been purported with yet unproven anti-inflammatory and antiCOVID-19 properties $^{32}$. No clinical evidence on the safety or efficacy of ruxolitinib, fedratinib, or any JAK inhibitor is available.

\section{Ibrutinib}

Ibrutinib an oral highly potent Bruton's tyrosine kinase (BTK) inhibitor used to treat indolent B-cell 
lymphoma and chronic graft-versus-host disease promotes the reduction of a pro-inflammatory cytokine profile frequently observed in these conditions ${ }^{36}$. The BTK pathway is also activated during infections, including viral pneumonia, mediating toll-like receptor signaling. Consequently, the BTK pathway is an attractive target for intervention in patients with severe COVID-1937. In an experimental influenza murine model, ibrutinib abrogated the development of lung injury and death, also showing a reduction in pro-inflammatory cytokine production ${ }^{38}$. A case series of 6 patients with Waldenström macroglobulinemia diagnosed with COVID-19 while receiving ibrutinib was recently reported. Five patients receiving $420 \mathrm{mg}$ required no hospitalization, and a patient receiving $140 \mathrm{mg}$ deteriorated shortly after stopping the drug, required mechanical ventilation, but improved rapidly after restarting and increasing the dose up to $420 \mathrm{mg}$. Albeit, this patient had also received several investigational treatments, including hydroxychloroquine, azithromycin, TCZ, and intravenous immunoglobulin. The authors suggested that hematological patients may benefit from continuing with ibrutinib as it may provide protection against lung inju$\mathrm{ry}^{38}$. Other frequent and potentially serious adverse effects associated to ibrutinib include atrial fibrillation and bleeding, particularly in patients receiving concurrent anti-aggregation or anticoagulation (such as critically ill COVID-19 patients) and also has been associated to an increased risk of Pneumocystis jiroveci pneumonia and invasive aspergillosis ${ }^{39}$. Similar to ruxolitinib, this drug is also financially toxic and unavailable to most persons living in LMICs. Registered clinical trials to examine the benefit of ibrutinib or other BTK inhibitors were not found in ClinicalTrials.gov at the time of writing. Therefore, the use of these drugs is not advisable at this time.

\section{Other proposed agents}

This sentence should read: Similarly to the new JAK and BTK inhibitors, older less expensive drugs used in hematology also have potential anti-COVID-19 efficacy, including but not limited to; tacrolimus, mycophenolate, mercaptopurine, vincristine, etoposide and anti-CD20 antibodes ${ }^{40}$. Although these drugs are certainly limited by a reduced interest of the pharmaceutical industry (among other funding sources) as they have mostly lost their patents, they would also merit careful scientific evaluation (Table 3). These less expensive and more easily available drugs represent an opportunity for performing meaningful clinical research in LMIC through carefully designed randomized clinical trials.

\section{Convalescent plasma therapy in patients with COVID-19}

Convalescent plasma therapy also known as passive immunization, consists of intravenous administration of plasma obtained from a patient who has recovered from infection and in whom it is possible to find immunoglobulin $M(\operatorname{lgM})$ and immunoglobulin $G(\lg G)$ antibodies against a certain microorganism, in this case against SARS-Cov-2, with the aim of neutralizing the virus and reducing the systemic inflammatory response (Table 3). The use of convalescent plasma has become more important during large-scale epidemics. The experience obtained in epidemics such as SARS and influenza pneumonia support its clinical utility. Patients who received this treatment had a shorter hospital stay and lower mortality, and no immediate adverse effect noted after plasma infusion ${ }^{41}$. A meta-analysis involving 1703 influenza pneumonia patients who received convalescent plasma showed reduced viral load and decreased in mortality by $21 \%{ }^{42}$.

Published clinical data on the usefulness of convalescent plasma in patients with COVID-19 are scarce without a randomized clinical trial yet available in this scenario. A systematic review included five studies with a total of 27 patients, most of them male residents of China, between 28 and 75 years old, 21 were in the intensive care unit, and 14 received mechanical ventilation. The plasma dose used was $80-2400 \mathrm{~mL}$, in 1-8 fractions, no serious adverse events were observed. All patients also received antiviral treatment. Although all studies were considered to have a risk of bias due to the heterogeneity of the studies and publication bias, mainly consisting of case reports and case series, it is relevant to note that all described an overall positive clinical result, with a significant reduction in viral load and an increase in neutralizing antibodies after treatment ${ }^{43}$. While convalescent plasma is feasible in many countries across the globe either through apheresis or whole blood donations in real time and can be frozen, several nuances must be highlighted. The ideal donor has not been identified, while identifying the presence of $\lg M$ and $\lg G$ antibodies against COVID-19 is feasible, identifying whether these antibodies are active against SARS-CoV2 is difficult and at the time of writing there is no commercially available assay. The appropriate dose and frequency of administration are unknown and convalescent plasma comes with the risk of allergic 
Table 3. Relevant treatment options from hematological point of view

The use of heparin should be strongly considered in high-risk
patients
Infusion of thrombolytic drugs has been used as a rescue
treatment albeit its safety is unconfirmed
Aspirin should be considered in patients with cardiac
dysfunction and increased troponin
Convalescent plasma therapy has been associated to a shorter
hospitalization and low mortality, confirmation of phase III trials
are pending
Tocilizumab has been used in severe COVID-19 with signs of CRS
Drugs with potential therapy in COVID-19
Ruxolitinib, fedratinib (JAK-2 inhibitors)
Ibrutinib (BTK inhibitor)
Tacrolimus
Mycophenolate
Mercaptopurine

CRS: cytokine release syndrome, JAK-2: Janus activating kinase, BTK: Bruton's tyrosine kinase

reactions and transfusion-associated circulatory overload, a problem that can limit appropriate ventilation.

In the Clinica Ruiz in Puebla, Mexico, a pilot study with convalescent plasma was performed from April to May in 10 severally ill male patients with COVID-19. The patients had a Kirby index below 300 , showing severe pulmonary injury. After receiving convalescent plasma from five donors, the patients were followed to evaluate the evolution of the disease.

The aim of this study was to state if this intervention could be an affordable and safe therapy for patients with COVID-19 in our country. However, even when the results were positive and demonstrated improvement in the disease course and respiratory function, the lack number of patients and the limitations this study has, cannot prove at all the effectiveness of this therapy ${ }^{44}$.

A recent systematic review evaluated 5443 patients of whom 5211 were treated with convalescent plasma. Some of the restrictions and adverse events include fever, allergic reactions, transfusion-related acute lung injury, and increased risk of thromboembolic events. The conclusions showed that the currently available evidence on the safety and effectiveness of convalescent plasma for the treatment of people hospitalized with COVID-19 is of a very low certainty ${ }^{45}$.

Randomized clinical trials are needed to define the efficacy of this therapy. However, with the information obtained so far, convalescent plasma could be a useful therapeutic option with promising evidence on safety. This kind of therapy could be considered a safe therapeutic option with promising effectivity. We must wait for more information in this setting, but nowadays, this therapy is already widespread.

\section{Conclusions}

The involvement of the hematologist in the COVID-19 pandemic is crucial. Many clinical, laboratory, and therapeutic aspects related to the hematology field are very important in dealing with hospitalized patients suffering from the disease. The amount of resources needed for the best treatment of COVID-19 patients is worrisome and the economic situation of LMICs is far from ideal. Several drugs such as TCZ, ruxolitinib, and ibrutinib are lacking in several countries or are expensive and out of reach for most health systems and patients. In this part of the world, we must be creative, optimize scarce resources, and search for the best local diagnostic and therapeutic approach.

\section{Conflicts of interest}

No financial conflicts of interest to disclose.

\section{Funding}

This study was locally funded.

\section{Ethical responsibilities}

Protection of people and animals. The authors declare that no human or animal experiments have been performed for this research.

Confidentiality of the data. The authors declare that they have followed the protocols of their workplace regarding the publication of patient data.

Right to privacy and informed consent. The authors declare that no patient data appear in this article.

\section{References}

1. Zhang L, Liu Y. Potential interventions for novel coronavirus in China: a systematic review. J Med Virol. 2020;92:479-90.

2. Singhal T. A review of coronavirus disease-2019 (COVID-19). Indian J Pediatr. 2020;87:281-6.

3. Liu Y, Yang Y, Zhang C, Huang F, Wang F, Yuan J, et al. Clinical and biochemical indexes from 2019-nCoV infected patients linked to viral loads and lung injury. Sci China Life Sci. 2020;63:364-74.

4. Huang C, Wang Y, Li X, Ren L, Zhao J, Hu Y, et al. Clinical features of patients infected with 2019 novel coronavirus in Wuhan, China. Lancet J. 2020;395:497-506.

5. Henry B, De Oliveira M, Benoit S, Plebani M. Hematologic, biochemical and immune biomarker abnormalities associated with severe illness and mortality in coronavirus disease 2019 (COVID-19): a meta-analysis. Clin Chem Lab Med. 2020;58:1021-8.

6. Tang N, Li D, Wang X, Sun Z. Abnormal coagulation parameters are associated with poor prognosis in patients with novel coronavirus pneumonia. J Thromb Haemost. 2020;18:844-7.

7. Song JC, Wang G, Zhang W, Zhang Y, Li WQ, Zhou Z. Chinese expert consensus on diagnosis and treatment of coagulation dysfunction in COVID-19. Mil Med Res. 2020;7:19. 
8. Zhou F, Yu T, Du R, Fan G, Liu Y, Liu Z, et al. Clinical course and risk factors for mortality of adult inpatients with COVID-19 in Wuhan, China: a retrospective cohort study. Lancet. 2020;395:1054-62.

9. Li J, Li Y, Yang B, Wang H, Li L. Low-molecular-weight heparin treatment for acute lung injury/acute respiratory distress syndrome: a meta-analysis of randomized controlled trials. Int J Clin Exp Med. 2018;11:414-22.

10. Tang N, Bai H, Chen X, Gong J, Li D, Sun Z. Anticoagulant treatment is associated with decreased mortality in severe coronavirus disease 2019 patients with coagulopathy. J Thromb Haemost. 2020;18:1094-9.

11. Thachil J, Tang N, Gando S, Falanga A, Cattaneo M, Levi M, et al. ISTH interim guidance on recognition and management of coagulopathy in COVID-19. J Thromb Haemost. 2020;18:1023-6.

12. Moore HB, Barrett CD, Moore EE, McIntyre RC, Moore PK, Talmor DS et al. Is there a role for tissue plasminogen activator (tPA) as a nove treatment for refractory COVID-19 associated acute respiratory distress syndrome (ARDS)? J Trauma Acute Care Surg. 2020;88:1.

13. Ruan $Q$, Yang K, Wang W, Jiang $L$, Song J. Clinical predictors of mortality due to COVID-19 based on an analysis of data of 150 patients from Wuhan, China. Intensive Care Med. 2020;46:846-8.

14. Ming Z. Cytokine storm and immunomodulatory therapy in COVID-19: role of chloroquine and anti-IL-6 monoclonal antibodies. Int J Antimicrob Agents. 2020;55:105982.

15. Aziz M, Aziz M, Fatima R, Assaly R. Elevated interleukin-6 and severe COVID-19: a meta-analysis. J Med Virol. 2020. In press. doi: https://doi. org/10.1002/jmv.25948

16. Gabay C, Riek M, Hetland ML, Hauge EM, Pavelka K, Tomšič M, et al Effectiveness of tocilizumab with and without synthetic disease-modifying antirheumatic drugs in rheumatoid arthritis: results from a European collaborative study. Ann Rheum Dis. 2016;75:1336-42.

17. Bachanova V, Bishop M, Dahi P. CAR T cell therapy during the COVID-19 pandemic: management strategies and challenges. Curr Res Translat Med. 2020:68:111-8.

18. Xu X, Han M, Li T, Sun W. Effective treatment of severe COVID-19 patients with tocilizumab. Proc Natl Acad Sci U S A. 2020;117:10970-5.

19. Zhang X, Song K, Tong F, Fei M, Guo H, Lu Z, et al. First case of COVID-19 in a patient with multiple myeloma successfully treated with tocilizumab. Blood Adv. 2020;4:1307-10.

20. Luo P, Liu Y, Qiu L, Liu X, Liu D, Li J. Tocilizumab treatment in COVID-19: a single center experience. J Med Virol. 2020;92:814-8.

21. Azienda MC, Fatebenefratelli $O$, Milano $O$, Alessandro M, Asst $O$, Sacco F. Favorable changes of CT findings in a patient with COVID-19 pneumonia after treatment with tocilizumab. Diagn Interv Imaging 2020;101:323-4

22. Michot JM, Albiges L. Tocilizumab, an anti-IL-6 receptor antibody, to treat COVID-19-related respiratory failure: a case report. Ann Oncol. 2020;31:961-4.

23. Radbel J, Narayanan N, Chest PB. Use of tocilizumab for COVID-19 infection-induced cytokine release syndrome: a cautionary case report. Chest. 2020;158:e15-9.

24. Qiu T, Liang S, Dabbous M, Wang Y, Han R, Toumi M. Chinese Guidelines related to Novel Coronavirus Pneumonia; 2020. Available from: http://www.preprints.org. [Last accessed on 2020 May 17].

25. Di Giambenedetto S, Ciccullo A, Borghetti A, Gambassi G, Landi F, Visconti $\mathrm{E}$, et al. Off-label use of tocilizumab in patients with SARS-CoV-2 infection. J Med Virol. 2020. In press. https://doi.org/10.1002/jmv.25897.

26. Genovese MC, McKay JD, Nasonov EL, Mysler EF, Da Silva NA Alecock E, et al. Interleukin-6 receptor inhibition with tocilizumab reduces disease activity in rheumatoid arthritis with inadequate response to disease-modifying antirheumatic drugs: the tocilizumab in combination with traditional disease-modifying antirheumatic drug therapy study. Arthritis Rheum. 2008;58:2968-80
27. Jones G, Sebba A, Gu J. Comparison of tocilizumab monotherapy versus methotrexate monotherapy in patients with moderate to severe rheumatoid arthritis: the AMBITION study. Ann Rheum Dis. 2010;69:88-96.

28. Kotch C, Barrett D, Teachey DT. Tocilizumab for the treatment of chimeric antigen receptor $\mathrm{T}$ cell-induced cytokine release syndrome. Expert Rev Clin Immunol. 2019;15:813-22.

29. Elli EM, Baratè C, Mendicino F, Palandri F, Palumbo GA. Mechanisms underlying the anti-inflammatory and immunosuppressive activity of ruxolitinib. Front Oncol. 2019;9:1186.

30. Kim E. Senotherapeutics: emerging strategy for healthy aging and age-related disease. BMB Rep. 2019;52:47-55.

31. Dillin A, Xu M, Palmer AK, Ding H, Weivoda MM, Pirtskhalava T, et al. Targeting senescent cells enhances adipogenesis and metabolic function in old age. Elife. 2015;4:e12997.

32. Stebbing J, Phelan A, Griffin I. COVID-19: combining antiviral and anti-inflammatory treatments. Lancet Infect Dis. 2020;20:400-2.

33. Zhang W, Zhao Y, Zhang F, Wang Q, Li T, Liu Z, et al. The use of anti-inflammatory drugs in the treatment of people with severe coronavirus disease 2019 (COVID-19): the experience of clinical immunologists from China. Clin Immunol. 2020;214:108393.

34. Wu D, Yang XO. TH17 responses in cytokine storm of COVID-19: an emerging target of JAK2 inhibitor fedratinib. J Microbiol Immunol Infect. 2020;53:368-70

35. Bewersdorf J, Jaszczur S. Beyond ruxolitinib: fedratinib and other emergent treatment options for myelofibrosis. Cancer Manag Res. 2019;11:10777-90.

36. Niemann CU, Herman SE, Maric I, Gomez-Rodriguez J, Biancotto A Chang BY, et al. Disruption of in vivo chronic lymphocytic leukemia tumor-microenvironment interactions by ibrutinib-findings from an investigator-initiated Phase II study. Clin Cancer Res. 2016;22:1572-82.

37. Treon SP, Castillo J, Skarbnik AP, Soumerai JD, Ghobrial IM, Guerrera $\mathrm{ML}$, et al. The BTK inhibitor ibrutinib may protect against pulmonary injury in COVID-19-infected patients. Blood. 2020;135:1912-5.

38. Florence JM, Krupa A, Booshehri LM, Davis SA, Matthay MA, Kurdowska AK. Inhibiting bruton's tyrosine kinase rescues mice from lethal influenza-induced acute lung injury. Am J Physiol Lung Cell Mol Physiol. 2018;315:L52-8

39. Paydas S. Management of adverse effects/toxicity of ibrutinib. Crit Rev Oncol Hematol. 2019;136:56-63.

40. Russell B, Moss C, George G, Santaolalla A. Associations between immune-suppressive and stimulating drugs and novel COVID-19-a systematic review of current evidence. Ecancermedicalscience. 2020;14:1022.

41. Liu W, Fontanet A, Zhang P, Zhan L, Xin Z, Baril L, et al. Two-year prospective study of the humoral immune response of patients with severe acute respiratory syndrome. J Infect Dis. 2006;193:792-5.

42. Luke T, Kilbane E, Jackson JL. Meta-analysis: convalescent blood products for Spanish influenza pneumonia: a future H5N1 treatment? Ann Intern Med. 2006;145:599-609.

43. Rajendran K, Narayanasamy K, Rangarajan J, Rathinam J, Natarajan M, Ramachandran A. Convalescent plasma transfusion for the treatment of COVID-19: systematic review. J Med Virol. 2020. In press. doi: https:// doi.org/10.1002/jmv.25961.

44. Olivares-Gazca JC, Priesca-Marín JM, Ojeda-Laguna M, Garces-Eisele J, Soto-Olvera S, Palacios-Alonso A, et al. Infusion of convalescent plasma is associated with clinical improvement in critically ill patients with COVID-19: a pilot study. Rev Invest Clin. 2020;72:159-64.

45. Piechotta V, Chai KL, Valk SJ, Doree C, Monsef I, Wood EM, et al. Convalescent plasma or hyperimmune immunoglobulin for people with $\mathrm{CO}$ VID-19: a living systematic review. Cochrane Database Syst Rev. 2020;7:CD013600 